\title{
Factores psicosociales de riesgo asociados a la conducta violenta en jóvenes salvadoreños
}

\section{Risk psychosocial factors related to violent behavior in Salvadorean youth}

\author{
José Ricardo Gutiérrez-Quintanilla \\ jose.gutierrez@utec.edu.sv \\ María Elizabet Campos-Tomasino² \\ Cecilia Beatriz Portillo-García ${ }^{3}$ \\ Investigadores Universidad Tecnológica de El Salvador
}

Recibido: 17/03/2015 - Aceptado: 16/06/2015

\section{Resumen}

El presente artículo es un extracto de un estudio mayor de tipo ex post facto, y tiene el objetivo de analizar los factores psicosociales de violencia asociados al comportamiento agresivo juvenil en El Salvador, para ello, se utilizó un diseño retrospectivo y transeccional, en una muestra probabilística de 3.349 jóvenes (1.708 mujeres y 1.640 hombres), con una media de edad total de 16.47 años (DT = 1.38). Los instrumentos de medida utilizados fueron los siguientes: la Escala de historia de violencia juvenil, Escala social y contextual de violencia juvenil, Escala de factores individuales de violencia juvenil, Escala de factores de protección de violencia juvenil y Cuestionario de agresión general. Los resultados revelan que los jóvenes con mayor riesgo de incurrir en conductas violentas son del sexo masculino, los residentes urbanos y los de centros educativos privados. Tanto los factores individuales de personalidad como los

\section{Abstract}

This article is an extract from an expos facto larger study whose aim is to analyze the psychosocial factors associated to aggressive youth behavior in El Salvador. In order to do this, a transactional and retrospective design was used in a probabilistic sample of 3.349 youths (1.708 were female and 1.640 were male). Their average age was 16.47 years old $(S D=1.38)$. The instruments used [to conduct the research] included the following: the juvenile violence history scale, the contextual and social scale of juvenile violence, the individual factors of youth violence scale, and the aggression questionnaire. The results revealed that the young people most at risk of incurring in violent behaviors are male urban residents who attend a private school. Both individual personality factors and social factors are significantly related to violent behavior in Salvadorean young people.

Doctor en Psicología. Profesor, investigador. Universidad Tecnológica de El Salvador. . Correo electrónico jose.gutierrez@utec.edu.sv.

Licenciada en Psicología, pasante de investigación en la Vicerrectoría de Investigación de la Universidad Tecnológica de El Salvador.Correo electrónico happy_4ever05@ hotmail.com

Licenciada en Psicología, pasante de investigación en la Vicerrectoría de Investigación de la Universidad Tecnológica de El Salvador. Correo electrónico cciliaportillo@gmail.com 
sociales están relacionados de forma significativa con la conducta violenta de los jóvenes salvadoreños.

\section{Palabras clave}

Factores de riesgo, violencia, agresión, jóvenes, El Salvador.

\section{Keywords}

Risk factors, violence, aggression, young people, El Salvador.
En el abordaje de la violencia juvenil es fundamental definir y comprender los factores asociados a la conducta violenta. En este sentido, es necesario destacar que existe bastante coincidencia entre los diferentes autores en demostrar que los factores psicosociales vinculados con el comportamiento violento de los jóvenes son de tipo personales o individuales, relacionales y sociales. El analizar estos factores solo tiene sentido en virtud de la prevención de la violencia. En la preparación del presente artículo surgieron las preguntas: ¿qué factores psicosociales de riesgo de violencia están asociados a la conducta violenta de los jóvenes salvadoreños?, ¿qué prevalencia de factores de riesgo de violencia y comportamientos violentos existen en los jóvenes?, ¿existen diferencias estadísticas en el comportamiento agresivo de los jóvenes en función de las variables sociodemográficas como el sexo, la residencia y el centro educativo? La estructura del presente informe sigue el orden siguiente: introducción, método, resultados, discusión y referencias. En los resultados de este artículo se tratará de responder las preguntas antes señaladas.

La población salvadoreña vive con la tasa de homicidios juveniles más alta del mundo (PNUD, 2013). El homicidio es la manifestación más extrema de la violencia. Sin embargo, los adolescentes y jóvenes constituyen, también, la población más afectada por diversos tipos de violencia, que incluyen el abuso físico, sexual, verbal y emocional, así como el abandono. Las posibilidades de que los jóvenes y adolescentes tengan un desarrollo sano se ven limitadas cuando son afectados por la violencia, ya sea como testigos, víctimas o agresores (OPS/OMS 2002). Los jóvenes son el grupo más afectado por la violencia; al igual que son los mayores responsables de ejercerla (PNUD, 2013).

El comportamiento violento cruza constantemente las fronteras entre el individuo, la familia, la comunidad y la sociedad (Malvaceda-Espinoza, 2009). A su vez, sus consecuencias abarcan estos ámbitos (Unicef, 2006). La violencia limita fuertemente las libertades y las capacidades de las personas (PNUD, 2013). Esto conlleva a la necesidad de conocer más y mejor sus causas, identificar los factores psicosociales que la previenen, la incitan y saber cuál es la magnitud de sus efectos. Tan pronto se invierta en la niñez y la juventud habrá más posibilidades de prevenir la violencia durante la edad adulta (Gonzáles, 2012). Los costos sociales y económicos de la violencia tienden a ser más altos que el costo efectivo de los programas, ya sea de prevención o reinserción de jóvenes victimarios o víctimas de violencia (Gonzáles, 2012).

Por tanto, son múltiples los factores psicosociales de riesgo que pueden estar relacionados con la situación de la violencia juvenil que atraviesa el país. Existen pocos estudios a escala nacional que analicen sistemáticamente los factores de riesgo de la violencia juvenil de forma integral. Ante este contexto, la Universidad Tecnológica de El Salvador (Utec) y la Policía Nacional Civil (PNC) firmaron un convenio de cooperación (2012) para implantar algunas estrategias concretas encaminadas al diseño de instrumentos, el diagnóstico de los factores de riesgo y protección de violencia juvenil, con la finalidad de incidir en la disminución o mejorar el contexto de violencia juvenil del país y sus consecuencias en la población. En este sentido, la Utec presentó, en el 2013, un estudio a escala nacional titulado "Evaluación de factores psicosociales de riesgo y de protección de violencia juvenil en El Salvador", con la finalidad de analizar un conjunto de factores psicosociales que predisponen a los jóvenes a la violencia, tanto siendo víctimas como agresores. Una de las tareas del estudio fue identificar los grupos etarios en riesgo de incurrir en conductas desviadas, como la agresión física o verbal, el consumo de drogas y alcohol, entre otros factores de riesgo. El presente artículo es un extracto de dicho estudio. 
El "Informe mundial sobre la violencia y la salud" preparado por la Organización Panamericana de la Salud y la Organización Mundial de la Salud (OMS), en el 2003, explica que Latinoamérica es la región que sufre el mayor impacto de la violencia en el mundo. Siendo los jóvenes el grupo más afectado por esta. La tasa promedio de homicidios de jóvenes de entre 15 y 29 años es de 101.7 por 100 mil hombres y de 11.5 por 100 mil mujeres. Se calcula que por cada asesinato hay entre 20 a 40 víctimas, adultas y jóvenes, involucradas en actos de violencia sin consecuencias mortales, pero que requieren atención intrahospitalaria (OMS, 2003). En casi todos los países de Latinoamérica se reconoce que la violencia que afecta a los jóvenes es un problema político y de salud pública. Su prevalencia no solamente tiene repercusiones en el desarrollo juvenil, sino también mina los fundamentos democráticos de la sociedad y es responsable por costos humanos, económicos y sociales enormes en la región. Los hechos de violencia explican la mayor parte de las muertes de los jóvenes en la región, especialmente tratándose de hombres de bajos ingresos, residentes en barrios degradados social y físicamente (OPS/OMS, 2006).

Kaleel, Justicia, Benítez y Pichardo (2007) realizaron una investigación en Palestina con una muestra de 1.492 alumnos de educación primaria y secundaria, cuyas edades oscilaban entre los 10 y 16 años, en centros educativos de la zona urbana y rural de Hebrón. Los resultados mostraron que el $53,5 \%$ de los participantes hombres informaron haber agredido a alguno de sus compañeros al menos una vez por semana, frente al $46,5 \%$ de mujeres igualmente agresoras. Las diferencias entre hombres y mujeres resultaron estadísticamente significativas. Así, los hombres que agreden a sus compañeros dos o tres veces por semana representan el $88 \%$ de los agresores. Por otra parte, el estudio sobre el comportamiento juvenil de Rochester se centró en las causas y consecuencias de la delincuencia juvenil y el consumo de drogas en una muestra de adolescentes urbanos calificados en una situación de alto riesgo de delincuencia y consumo de drogas $(\mathrm{n}=$ 1.000; 729 hombres y 271 mujeres) desde su temprana adolescencia (séptimo y octavo grados) hasta su juventud (Browning, Thornberry \& Porter, 1999; Lizotte \& Sheppard, 2001). El estudio examinó la relación de la delincuencia con variables familiares, escolares, de amistad y comunitarias, obteniéndose las siguientes conclusiones: los niños que reciben un mayor grado de cariño y compromiso de sus padres tienen menos implicación en actividades delictivas; un pobre rendimiento escolar se encuentra asociado a un incremento en implicaciones delictivas y de consumo de drogas; la relación con amigos delincuentes hace más fuerte y consistente la asociación a la delincuencia; y que los niños de clase baja tienen mayores implicaciones en conductas delincuentes (Browning, Thornberry \& Porter, 1999). La literatura criminológica más reciente (Seydlitz \& Jenkins, 1998; Leukefeld et al., 1998; Hawkins et al., 2000; Kazdin \& Buela-Casal, 2001; Wasserman et al., 2003) señala como ámbitos de factores de riesgo más influyentes en el comportamiento antisocial y delincuente de los jóvenes a la familia, la escuela, el grupo de amigos, el consumo de drogas y la comunidad.

En un trabajo de Goldstein (1990), al ser preguntada a una serie de delincuentes condenados por las causas que los llevaron al delito, mencionaron un mayor número de veces la familia, seguido de las influencias de los amigos y las drogas; además de la escuela y aspectos comunitarios como la pobreza (citado por Seydlitz \& Jenkins, 1998). Junto con la familia, la escuela aparece como un factor determinante en la correcta educación y socialización de los jóvenes, operando como un inhibidor de la delincuencia, ya que el éxito académico y buenas actitudes hacia la escuela reducen la delincuencia (Seydlitz \&Jenkins, 1998; Browning \& Huizinga, 1999; Parks, 2000; Schweinhart, 2003; Burns, Howell et al., 2003), aunque en algunos casos el fracaso escolar o un temprano abandono escolar opera en sentido contrario como un facilitador de la delincuencia (Farrington, 1992, 1997; Borduin \& Schaeffer, 1998; Catalano, Loeber \& McKinney, 1999; Huizinga, Loeber, Thornberry \& Cothern, 2000). En estudio realizado con adolescentes colombianos sobre los factores de riesgo de violencia, Brook, Brook, Zohn, De la Rosa, Montoya y Whiteman (2003) encontraron que los factores de riesgo hacia la violencia juvenil eran la personalidad del adolescente y los atributos de comportamiento (ejemplos: consumo de drogas y la tolerancia); las características de la familia, tales como el uso de drogas en los padres y hermanos, conflictos padre-hijo; factores de pares, tales como el consumo de drogas entre pares y la desviación en los pares; y factores ecológicos, que incluyen la disponibilidad de drogas y la prevalencia de la violencia en la comunidad. Los factores de riesgo ejercen 
una mayor o menor influencia, dependiendo de la edad en la que aparecen (Farrington, 1992; Lipsey \& Derzon, 1998; Hawkins et al., 2000); existe una mayor incidencia de conductas antisociales y delictivas en los jóvenes de género masculino. En este mismo sentido, Murcia, Reyes, Gómez, Medina, Paz y Fonseca (2007), en su estudio realizado en Honduras con una muestra de 576 escolares de ambos sexos, cuyas edades fluctuaban entre los $10 \mathrm{y}$ 15 años, procedentes de escuelas públicas y privadas, a quienes se aplicó el Inventario de hostilidad de Buss y Burke y una entrevista familiar semiestructurada, los resultados mostraron que los niños con mayores niveles de hostilidad y agresividad proceden de hogares desintegrados y disfuncionales, con evidencias de violencia doméstica y alcoholismo. En los estudios anteriores se pueden apreciar claramente algunos de los efectos psicológicos y emocionales que sufren los jóvenes por la falta de una figura paterna, y sus consecuencias en el desarrollo normal de la personalidad como el autoconcepto, el control emocional, la conducta violenta y el consumo de drogas y alcohol. Configurándose los problemas antes señalados como factores de riesgo de violencia juvenil.

Igualmente, en El Salvador, Gutiérrez, Sánchez y Martínez (2013) encontraron que los jóvenes que presentan una mayor prevalencia de ambiente familiar disfuncional y conductas desviadas son aquellos que han crecido con una solo figura paterna. Entre los problemas de mayor incidencia encontrados se tienen los siguientes: que son más víctimas de violencia psicológica, presentan menos supervisión y control, viven en un ambiente familiar hostil, son víctimas de violencia física y presentan más inestabilidad emocional que los jóvenes que crecieron con ambos padres. zúñiga y Bermúdez (2010) encontraron, en jóvenes salvadoreños, que quienes habían crecido en hogares desintegrados presentaban mayores problemas en el autoconcepto académico, personal y emocional; tenían más ansiedad, inseguridad, estrés y problemas de relaciones interpersonales que los jóvenes que vivían con ambos padres. Estudio reciente encontró que $47,5 \%$ de los jóvenes salvadoreños viven con un solo padre o pariente cercano (Gutiérrez, 2012). Al analizar el contexto familiar salvadoreño se encontró que más del $44 \%$ de los jóvenes de educación media viven con uno de sus padres (Orantes, 2012). Lo anterior indica una relación existente entre la desintegración familiar con las conductas desviadas de los jóvenes, como problemas de personalidad antisocial, de salud mental y psicosociales, consumo de drogas y alcohol, y conductas delictivas. También se muestra una relación de la conducta violenta con la dinámica familiar (maltrato infantil y juvenil) en los hogares salvadoreños.

Hawkins, Lishner, y Catalano (1985) consideran como factores de riesgo a "cualquier circunstancia o evento de naturaleza biológica, psicológica o social, cuya presencia o ausencia modifica la probabilidad de que se presente un problema determinado en una persona o comunidad". La violencia juvenil no puede ser explicada por factores aislados debido a que es el resultado de un sistema, por tanto, se considera importante el aporte de Bronfrenbrenner (1979), quien afirma que "la violencia es el resultado de la acción recíproca de factores individuales, relacionales, comunitarios, sociales y temporales" (enfoque ecológico), perspectiva asumida por la OMS (2003).

Mediante modelos explicativos, se han identificado tres grupos de factores de riesgo de la violencia (Laespada, Logan \& Arostegi, 2004). Entre estos factores se tienen los personales, relacionales y sociales. Los factores de riesgos individuales o personales hacen referencia a las características internas del individuo, a su forma de ser, sentirse y comportarse. La edad, la personalidad, las habilidades sociales de que dispone (Pons \& Berjano, 1999; Smith \& Scott, 1993); las actitudes, los valores, creencias hacia las drogas, la autoestima (Kaplan, 1996; Mendoza, Carrasco \& Sánchez, 2003) se han manifestado también como predictores fiables de la conducta adictiva (Fishbei \&Ajzen, 1980; Romero, 1996; Catalano \& Hawkins, 1996). A su vez, estos valores, creencias y actitudes vienen determinados, en primera instancia, por la familia como su contexto portador y transmisor (Ferrer \& Ayneto, 1991) y por la presión del grupo de iguales (GómezReino, Ferreiro, Domínguez \& Rodríguez, 1995); sobre la capacidad de tomar decisiones propias (Boys et al. 1999). Estos estudios llegan a resultados concluyentes afirmando que la realización de la conducta problema dependerá, en última instancia, de un proceso de toma de decisiones, de una valoración personal de pros y contras, beneficios y costes que pueden empujar a expresar dichas conductas o no manifestarlas. 
Por tanto, una eficaz estrategia de prevención de comportamientos antisociales en los jóvenes parte de identificar los factores de riesgo que influyen en esos comportamientos. Tanto las características individuales (de personalidad) como los contextos familiar, relacional, comunitario, social y las condiciones estructurales donde se desarrolla y convive el joven pueden ser calificados como factores de riesgo. Dependerá de la estabilidad, el equilibrio, la armonía y los valores de cada ámbito implicado. Lo anterior indica que los desajustes, la inestabilidad, la falta de control y armonía en cada dimensión finalizará convirtiéndose en un factor de riesgo de las conductas desviadas, como la agresividad, el consumo de drogas y alcohol; alteraciones de la personalidad como las conductas antisociales, inestabilidad emocional, la ansiedad, la depresión y actos delictivos. Si los factores de riesgo pueden ser disminuidos y los factores protectores aumentados por una acción preventiva, entonces las probabilidades de reducir la delincuencia y la violencia juvenil se verá incrementada (Howell, 1997; Hawkins et al., 2000).

El presente artículo pretende analizar algunos factores psicosociales de riesgo asociados a la conducta violenta juvenil salvadoreña; ante los potenciales efectos psicológicos y emocionales de la violencia juvenil en la población salvadoreña y de cara al diseño e implantación de programas de prevención primaria, secundaria y terciaria de la violencia juvenil del país. Se tienen los objetivos específicos siguientes: 1) identificar la prevalencia de factores psicosociales de riesgo de violencia juvenil, 2) establecer si existe correlación entre los factores psicosociales de riesgo con el comportamiento agresivo de los jóvenes salvadoreños, y 3) determinar si existen diferencias estadísticas significativas entre el comportamiento agresivo de los jóvenes en función de las variables sociodemográficas como el sexo, la residencia (urbana, rural) y los centros educativos del país a los que asisten dichos jóvenes.

\section{Método}

\section{Participantes}

En el presente estudio se seleccionó una muestra probabilística representativa de las ciudades más importantes del país. Para ello, se adoptó una estimación de error del 1,9\% y un nivel de confianza del $97 \%$, obteniéndose una muestra de 3.349 jóvenes de ambos sexos a escala nacional. La muestra fue distribuida de forma proporcional al número de estudiantes de educación media en cada departamento. En el estudio se aplicó un diseño retrospectivo y transeccional, utilizando como técnica de estudio la encuesta, mediante la evaluación colectiva. Es un estudio de tipo ex post facto (Montero \& León, 2007). En el estudio fueron evaluados $1.708(51,0 \%)$ mujeres y 1.640 $(49,0 \%)$ hombres; la media de edad total fue de 16.47 años $(D T=1.38)$; para las mujeres fue de 16.38 años $(D T=1.33)$ y para los hombres de 16.56 años $(D T=1.43)$. Se presenta una descripción de las variables sociodemográficas, como sexo, lugar de residencia, centro educativo, con quién vive, entre otras.

\section{Instrumentos}

Cuestionario de elaboración propia. Este apartado fue incluido en la primera página de la batería de pruebas y contiene todas las variables sociodemográficas, como sexo, edad, centro educativo, lugar de residencia, nivel educativo, con quién vive, departamento, si trabaja o no y actividad laboral.

Escala de historia de violencia juvenil (victimización) (Eshivi). Es una prueba que evalúa las experiencias pasadas y presentes de violencia en el hogar durante la niñez y la adolescencia; los supuestos teóricos están basados en el modelo de factores de riesgo de violencia juvenil planteado por Borum, Bartel y Forth (2003); la escala fue construida y validada por Gutiérrez, Sánchez y Martínez (2013); la prueba está estructurada en seis factores. Cada factor está conformado por los ítems como sigue: el factor 1, Víctima de violencia psicológica (ítems: 9, 10, 11, 12, 13, 14, 15, $16,18,19$ y 20$)$, con una consistencia interna mediante el alfa de Cronbach de 0.90; el factor 2, Supervisión y control de los padres (ítems: 24, 25, 26, 27, 28, 29 y 30), con una consistencia interna mediante el alfa de Cronbach de 0.78 ; el factor 3, víctima de violencia física (ítems: 5, 6, 7, 8 y 17), en el que la consistencia interna mediante el alfa de Cronbach fue de 0.73; el factor 4, Violencia física expresada (ítems: 1 , 2,3 y 4), en este la consistencia interna mediante el alfa de Cronbach fue de 0.67; el factor 5, Ambiente familiar hostil (ítems: 21, 22 y 23), con un alfa de Cronbach de 0.76; y el 
factor 6, Conflicto emocional (ítems: 31 y 32), con un alfa de Cronbach de 0.69. Tanto el análisis factorial como el de consistencia interna proporcionaron resultados aceptables en una muestra de adolescentes salvadoreños para medir factores históricos de violencia en el hogar.

Escala social y contextual de violencia juvenil. Es una prueba que evalúa algunos factores sociales y comunitarios durante la niñez y la adolescencia, que pueden incidir 0 empujar al joven al cometimiento de conductas desviadas como la violencia. La prueba está basada en los supuestos teóricos del modelo de factores de riesgo de violencia juvenil planteados por Borum, Bartel y Forth (2003); la escala fue construida y validada por Gutiérrez, Sánchez y Martínez (2013); la prueba está estructurada en los siguientes cuatro factores: estrés social, conducta desviada de los pares, apoyo social y resolución de problemas. Cada factor está conformado por los ítems como sigue: el factor 1, Estrés social (ítems: 7, 8, 9, 10, 12 y 14), con una consistencia interna mediante el alfa de Cronbach de 0.72; el factor 2, Conducta desviada de los pares (ítems: 1, 2, 3, 4, 5 y 6), con una consistencia interna mediante el alfa de Cronbach de 0.73; el factor 3, Apoyo personal (ítems: 16, 17 y 18), la consistencia interna mediante el alfa de Cronbach fue de 0.64, y el factor 4, Resolución de problemas (ítems: 11, 13, 15,19 y 21), la consistencia interna mediante el alfa de Cronbach fue de 0.64 . Tanto el análisis factorial como el de consistencia interna proporcionaron resultados aceptables en una muestra de adolescentes salvadoreños para medir factores sociales y contextuales de riesgo de violencia juvenil.

Escala de factores individuales de violencia juvenil. Es una prueba que evalúa factores individuales y personales durante la niñez y la adolescencia, que pueden empujar al joven al cometimiento de conductas desviadas como la violencia (delictiva). La prueba está basada en los supuestos teóricos del modelo de factores de riesgo de violencia juvenil planteados por Borum, Bartel y Forth (2003); la escala fue construida y validada por Gutiérrez, Sánchez y Martínez (2013). Cada factor está conformado por los ítems como sigue: el factor 1, Consumo de bebidas alcohólicas (ítems: 21, 22,13 y 24), con una consistencia interna mediante el alfa de Cronbach de 0.90; el factor 2, Actitudes negativas (ítems: 1, $2,3,4,8$ y 15), con una consistencia interna mediante el alfa de Cronbach de 0.84 ; el factor 3, Compromiso con la escuela (ítems: 37, 38, 39, 40, 41 y 42), en el que la consistencia interna mediante el alfa de Cronbach fue de 0.81 ; el factor 4, Rendimiento académico (ítems: 32, 33, 34, 35, 36 y 43), que su consistencia interna mediante el alfa de Cronbach es de 0.79; el factor 5, Empatía (ítems: 10, 11, 12, 13 y 14), su consistencia interna alfa de Cronbach es de 0.71; el factor 6, Manejo de la ira (ítems: $5,6,7,8$ y 9), en el que su alfa de Cronbach es de 0.73; el factor 7, Consumo de drogas ilícitas (ítems: 25, 26 y 27), en este el alfa de Cronbach es de 0.92; el factor 8, Consumo de cigarrillo (ítems: 17, 18, 19 y 20), con un alfa es de 0.81; y el factor 9, Daño autoinfligido (ítems:28, 29, 30 y 13), con un alfa de Cronbach de 0.72 .

Escala de factores de protección de violencia juvenil. Es una prueba que evalúa algunos factores de protección de violencia juvenil, que protegen al joven del cometimiento de conductas desviadas como la violencia (delictiva). La prueba está basada en los supuestos teóricos del modelo de factores de riesgo y protección de violencia juvenil planteados por Borum, Bartel y Forth (2003); la escala fue construida y validada por Gutiérrez, Sánchez y Martínez (2013); cada factor está conformado por los ítems como sigue: el factor 1, Compromiso con el rendimiento escolar (ítems: 6, 7, 8, 9, 10 y 11), con una consistencia interna mediante el alfa de Cronbach de 0.77 ; el factor 2, Acciones prosociales (ítems: 1, 2, 3, 4 y 5), con una consistencia interna mediante el alfa de Cronbach de 0.75; el factor 3, Modelaje de los padres (ítems: 12, 13 y 14), en este la consistencia interna mediante el alfa de Cronbach fue de 0.80; y el factor 4, Personalidad resiliente (ítems: 15, 16, 17, 18, 19, 20, 21 y 22), cuya consistencia interna mediante el alfa de Cronbach es de 0.79-

Cuestionario de Agresión General (Aggression Questionnaire, AQ) (Buss y Perry, 1992). Variables como la agresividad, la ira o la hostilidad forman parte de diversos modelos explicativos empleados tanto en la psicología clínica y de la salud como en la medicina conductual; este es el caso, por ejemplo, de la conducta antisocial (Ireland y Archer, 2004; Morren y Meesters, 2002; Palmer y Thakordas, 2005), la violencia conyugal (Echeburúa y Fernández, 1998) y la violencia escolar (Lawrence y Green, 2005). La versión original del Cuestionario de Agresión de Buss y Perry (1992) quedó conformada por cuatro factores (agresión física, 
agresión verbal, ira y hostilidad), los que mostraron una fiabilidad de consistencia interna aceptable, situándose entre 0.72 y 0.85 , dependiendo de la subescala. Esta prueba ha sido adaptada en diferentes culturas y países. Sierra y Gutiérrez (2007) realizaron un estudio de adaptación del cuestionario en estudiantes universitarios salvadoreños, obteniendo evidencias empíricas similares a los estudios anteriores. El análisis factorial confirmó la estructura tretrafactorial, eliminándose los ítems 3 y 24 por tener una carga factorial baja, quedando la versión salvadoreña con 27 ítems; también se obtuvieron los índices de consistencia interna, mediante el alfa de Cronbach. Estos se describen a continuación: Agresión física ( $a=0.80)$, ítems: $1,4,8,12,16$, 20, 25 y 27; Agresión verbal ( $a=0.73)$, ítems: $2,5,6,9,10$ y 13; Hostilidad ( $\mathrm{a}=0.76)$, ítems: 3,7, 11, 15, 19, 22, 24 y 26; Ira $(\mathrm{a}=0.72)$ ítems: 14, 17, 18, 21 y 23.

\section{Procedimiento}

Tras la construcción y validación de los instrumentos en la fase uno del estudio, se procedió a la elaboración del cronograma del trabajo de campo que contenía las ciudades, fechas y horas de salida a las distintas ciudades del país. Posteriormente se realizó una capacitación a los coordinadores de departamento y los equipos de evaluadores, con relación a las distintas fases y etapas del proceso de recolección de datos. También se explicó el formato de la carta solicitud que fue enviada a los directores de los complejos educativos e institutos nacionales que fueron seleccionados por los coordinadores. Así mismo, se explicó el perfil que debía cumplir el centro escolar. Previo a cada día de salida, la PNC ponía a disposición el transporte para el equipo evaluador. Según el calendario del trabajo de campo, fueron seleccionados los miércoles y viernes para visitar los complejos educativos en cada ciudad. Esta fase de recolección de datos inició el 08 de mayo y finalizó el 10 de julio de 2013. Antes de cada salida, el equipo evaluador se reunía entre las 06:00 y 07:30 a.m. en las instalaciones de la Utec para trasladarse al departamento correspondiente, según la planificación. Al llegar a cada ciudad, se hacía contacto con el coordinador in situ, y este orientaba al equipo evaluador acerca de los centros seleccionados. Estando en el centro educativo, el coordinador de departamento hacía el contacto con el director para determinar las secciones 0 grupos de clases que se debían evaluar; en este momento se designaban los subgrupos de evaluación para cada sección; se entregaban a los subgrupos de evaluación el número de pruebas que debían administrar, según el número de estudiantes en cada sección. Al momento de abordar a los estudiantes, los evaluadores cumplían la siguiente rutina: presentación del equipo, exposición del propósito de la visita, breve explicación del proyecto y solicitud de colaboración para responder el cuestionario. Se les leía la parte introductoria y la forma de contestar el instrumento. También se insistía en la importancia del estudio, de la anonimidad y de la sinceridad en la respuesta.

\section{Resultados}

El análisis de los resultados del presente artículo se presenta en el orden de sus objetivos. En un primer momento, se presentan los análisis descriptivos que permiten identificar la prevalencia de los factores psicosociales de riesgo de violencia en los jóvenes salvadoreños. Para este primer análisis, los factores de riesgo de violencia juvenil fueron agrupados en tres niveles de percentil: bajo la media, en la media y superior a la media, indicando en la mayoría de los factores evaluados que los valores superiores a la media tienen una interpretación negativa (- es negativo), los valores bajo la media son interpretados como positivos (+ es positivo), mientras que los que están en la media son interpretados como normales o positivos. Sin embargo, en el factor Supervisión y control de los padres, los valores altos tienen un significado positivo y los bajos un sentido negativo. A continuación se presentan las frecuencias y porcentajes negativos de los factores psicosociales de riesgo de violencia juvenil.

Los resultados revelan que 1.222 (38,2 \%) de los(as) jóvenes son víctimas de violencia psicológica en su familia; 1.129 $(35,1 \%)$ no reciben una adecuada supervisión y control de los padres; también se encontró que 1.217 (36,9 \%) han sido víctimas de violencia física en la niñez y la adolescencia; $1.442(43,7 \%)$ de los(as) jóvenes viven en un ambiente familiar hostil; $1.243(38,2 \%$ ) jóvenes presentan estrés social; se encontró que 1.509 (46,0 \%) tienen amigos o compañeros con una conducta desviada; en cuanto al consumo de bebidas alcohólicas, 877 (26,4 \%) presentan esta conducta; 1.209 (36,5 \%) tienen una actitud negativa; 1.357 (42,2 \%) de los jóvenes presentan un bajo rendimiento académico. 
Mientras que en los factores de conducta agresiva, se tiene que 1.454 (45,2 \%) presentan agresión física; 1.433 $(44,1 \%)$ presentan agresión verbal; 1.384 (42,9%) tienen rasgos de personalidad de hostilidad, característica básica en la conducta agresiva; y $1.805(33,1 \%)$ presentan la característica de ira, que tiene una correlación significativa positiva con la hostilidad, con la agresión física y verbal (ver tabla 1).

Tabla 1.

Frecuencias y porcentajes de prevalencias de factores psicosociales de riesgo de violencia y comportamientos agresivos en adolescentes salvadoreños

\begin{tabular}{lcc}
\hline \multicolumn{1}{c}{ VARIABLES } & $\mathrm{n}$ & $\%$ \\
\hline Factores de riesgo de violencia & & \\
Víctima de violencia psicológica & 1.222 & 38,2 \\
Supervisión y control de los padres & 1.129 & 35,1 \\
Víctima de violencia física & 1.217 & 36,9 \\
Ambiente familiar hostil & 1.442 & 43,7 \\
Conflicto emocional & 1.622 & 49,2 \\
Estrés social & 1.243 & 38,2 \\
Conducta desviada de los pares & 1.509 & 46,0 \\
Consumo bebidas alcohólicas & 877 & 26,4 \\
Actitudes negativas & 1.209 & 36,5 \\
Compromiso con la escuela & 1.433 & 44,7 \\
Rendimiento académico escolar & 1.357 & 42,2 \\
Manejo de la ira & 1.597 & 48,1 \\
Consumo de drogas ilícitas & 422 & 12,70 \\
Consumo de cigarrillos & 897 & 27,4 \\
Compromiso con el rendimiento escolar & 1.437 & 44,3 \\
Personalidad resiliente & 1.300 & 39,9 \\
Violencia física expresada & 1.437 \\
Agresión física & 1.218 & 37,4 \\
Agresión verbal & 1.805 & 45,2 \\
Ira & & 44,1 \\
\hline
\end{tabular}




\section{Diferencia de medias y correlaciones}

En el análisis de comparación de medias de la conducta agresiva en función de algunas variables sociodemográficas se aplicó la prueba $t$ de student, para muestras independientes. En Agresión física, en función del sexo, se encontró que existen diferencias estadísticamente significativas entre hombres y mujeres $\left(t_{3217}=-13.614 ; p\right.$ $<$.001), presentando mayor índice de agresión física los hombres en comparación con las mujeres. Al comparar la misma variable (Agresión física) en función del centro educativo, se encontró que existen diferencias significativas entre los centros públicos y los privados $\left(t_{3198}=-2.357\right.$; $\mathrm{p}=.018$ ), indicando que existe una mayor prevalencia de agresión física en los jóvenes de centros educativos privados que en los públicos; no se encontraron diferencias significativas en función del lugar de residencia (urbano y rural). Al analizar la variable Agresión verbal, en función del centro educativo, se encontró diferencias significativas entre centros públicos y privados $\left(t_{3233}=-4.279 ; p<.001\right.$ ), reflejando que presentan más conductas de agresión verbal los jóvenes de centros educativos privados en contraste con los de centros públicos; no se encontraron diferencias estadísticamente significativas entre hombre y mujeres, ni entre jóvenes rurales y urbanos. En la variable Hostilidad, en función del sexo, se encontró que existen diferencias significativas entre hombres y mujeres (t3226 $=5.914$; $\mathrm{p}<.001$ ), presentando mayor hostilidad las mujeres en comparación con los hombres; no existen diferencias significativas en función del lugar de residencia y del centro educativo. En la variable Ira no existen diferencias significativas en función del sexo, lugar de residencia o centro educativo. Al analizar la variable violencia física expresada, en función del sexo, se encontró que existen diferencias estadísticas significativas entre hombres y mujeres $\left(t_{3256}=-5.656 ; p<0.001\right)$, encontrándose mayor expresión de violencia física en los hombres en comparación con las mujeres; no se encontraron diferencias significativas en esta variable con relación al lugar de residencia ni al centro educativo.

Aplicando las correlaciones de Pearson (r) entre algunos factores psicosociales de riesgo de violencia juvenil y entre algunos comportamientos agresivos, se observaron las siguientes correlaciones: que existe una correlación estadística significativa entre víctima de violencia física y víctima de violencia psicológica $(r=.582 ; p<01)$; entre ambiente familiar hostil y víctima de violencia psicológica $(r=.578 ; p<01)$; entre actitudes negativas y hostilidad $(r$ $=.498 ; \mathrm{p}<.01)$; entre manejo de la ira y agresión física $(r=$ .487; $p<.01)$; entre manejo de la ira y agresión verbal $(r=$ $500 ; \mathrm{p}=<.01)$ (ver tabla 2). 


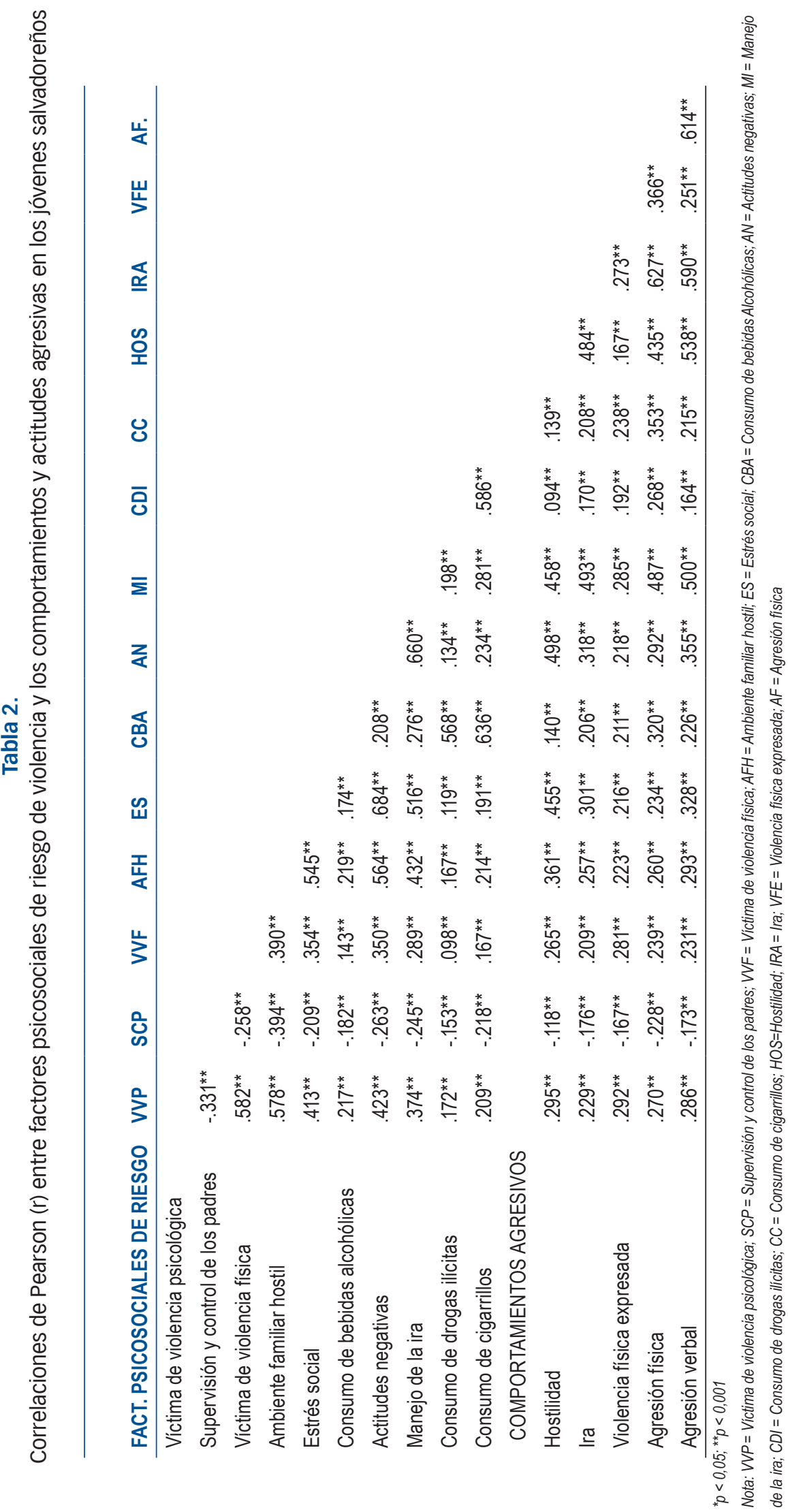




\section{Discusión}

El fenómeno de la conducta violenta es un problema complejo, multicausal, multidimensional y multifactorial, que ha sido estudiado a través de los años. Esta conducta es abordada por diferentes disciplinas, como la psicología, psiquiatría, sociología, antropología, criminología, biología, neurología, medicina y el derecho, entre otras. La presente discusión está centrada en poner de relieve aquellos factores de riesgo más relevantes y que, en estudios previos, han demostrado su relación con los comportamientos violentos del ser humano. No podemos obviar decir que este estudio se realizó desde la perspectiva de la psicología con implicaciones y aplicaciones en diferentes disciplinas. La conducta violenta es tipificada universalmente como una conducta desviada, es decir, una conducta que no se ajusta a las expectativas y las normas socialmente aceptadas. Una de las definiciones generales de la violencia dice que "es toda acción que tiene la intención de producir daño a la persona". Existen diferentes formas y tipos de violencia: autoinfligida, interpersonal y colectiva-estructural, cada una con distintos subtipos, cuya naturaleza es diferente; puede ser física, psicológica, sexual, por negligencia, abandono u omisión (OMS, 2003), lo que significa que no se pueden generalizar los actos violentos como si fueran iguales. Entre Ios factores de riesgo de violencia juvenil en El Salvador fueron analizadas las siguientes variables: víctima de violencia psicológica, supervisión y control de los padres, víctima de violencia física, ambiente familiar hostil, conflicto emocional, estrés social, conducta desviada de los pares, apoyo social, resolución de problemas, consumo de bebidas alcohólicas, compromiso con la escuela, rendimiento académico, consumo de cigarrillos, agresión física, agresión verbal y hostilidad.

También, Browning et al., 1999; Browning, Thornberry \& Porter, 1999; Lizotte \& Sheppard, 2001, en sus estudios, examinaron la relación de la delincuencia con variables familiares, escolares, de amistad y comunitarias, obteniéndose las siguientes conclusiones: los niños que reciben un mayor grado de cariño y compromiso de sus padres tienen menos implicación en actividades delictivas; un pobre rendimiento escolar se encuentra asociado con un incremento en implicaciones delictivas y de consumo de drogas; la relación con amigos delincuentes hace más fuerte y consistente la asociación con la delincuencia; y que los niños de clase baja tienen mayores implicaciones con conductas delincuentes (Browning, Thornberry \& Porter, 1999).

El análisis del factor Víctima de violencia psicológica en la infancia revela que existen cerca de cuatro de cada diez jóvenes salvadoreños que fueron víctimas de este maltrato en el hogar; en el factor Supervisión y control de los padres, se observa que más de tres de cada diez jóvenes no reciben supervisión de los padres; referente al factor Víctima de violencia física, el estudio revela que cerca de cuatro de cada diez jóvenes son víctimas de esta violencia; al hablar del comportamiento agresivo, se hace referencia a una multicausalidad. Tal comportamiento está influenciado por diferentes factores: sociales, culturales, genéticos y biológicos, tanto del ámbito familiar como del individual, justificándose así el abordaje desde diversos enfoques teóricos (Castrillón, Ortiz, \& Vieco, 2004; citados por Castillo, 2006); en el factor Ambiente familiar hostil se observó que más de cuatro de cada diez jóvenes presentan hostilidad en su familia; en Conducta desviada de los pares, el estudio revela que cerca de la mitad de los(as) jóvenes tienen amigos que expresan alguna conducta desviada; el grupo de amigos (peers) es importante en el desarrollo psicosocial al ofrecer a los adolescentes un sentido de pertenencia, un soporte emocional y normas de comportamiento (Borduin \& Schaeffer, 1998), señalándose como un factor de gran influencia en la delincuencia juvenil, hasta llegar a afirmarse que la asociación con amigos delincuentes es el mejor predictor de la delincuencia en las investigaciones actuales (Howell, 1997; Battin-Pearson, Thornberry, Hawkins \& Krohn, 1998; Lawrence, 1998; Seydlitz \& Jenkins, 1998; Leukefeld et al., 1998; Borduin \& Schaeffer, 1998; Browning \& Huizinga, 1999; Feld, 1999; Browning, Thornberry \& Porter, 1999; Hawkins et al., 2000; Wasserman et al., 2003); con relación al consumo de bebidas alcohólicas, el estudio revela que cerca de un tercio de los jóvenes consumen alcohol, entre los factores de riesgo hacia la violencia en adolescentes son coincidentes los enfoques planteados por Luengo-Martín y Tavares-Filho (1997) y Moncada-Bueno (1997), quienes señalan que existen factores de riesgo hacia la violencia y el consumo de drogas, los cuales pueden tipificarse como factores individuales, micro y macrosociales. En Consumo de cigarrillos, se encontró que más de un cuarto de los(as) 
jóvenes consumen cigarrillos. Existen evidencias empíricas que apoyan una fuerte relación entre el consumo de drogas y la delincuencia. Huizinga, Loeber, Thornberry y Cothern (2000) sostienen que los factores de riesgo que contribuyen al consumo de drogas son los mismos o muy similares a los que influyen en la delincuencia (Hawkins, Arthur y Catalano, 1995; Leukefeld et al., 1998); en el factor Rendimiento académico, el estudio revela que más de cuatro de cada diez jóvenes presentan un bajo rendimiento académico; en el factor Violencia física expresada, el estudio revela que cerca de cuatro personas de cada diez presentan un comportamiento de agresión física; en la variable Agresión física, cerca de la mitad de los(as) jóvenes presenta una conducta agresiva. Dentro de este grupo, los más afectados son los hombres y los jóvenes de centros educativos privados. Los hallazgos en Agresión verbal son similares a los de agresión física, indicado que existe una correlación muy alta entre estas dos modalidades de agresión. También existe una correlación importante entre los factores de riesgo de violencia y los comportamientos agresivos, evidenciándose empíricamente que tanto los factores individuales de personalidad como los sociales: la familia, la comunidad, los amigos, están relacionados de forma significativa y positiva con la conducta agresiva de los jóvenes salvadoreños.

\section{Referencias}

Battin-Pearson, S.R.; Thornberry, T.P.; Hawkins, J.D., \& Krohn, M. D., (1998). "Gang Membership, Delinquent Peers, and Delinquent Behavior", en Juvenile Justice Bulletin, October, OJJDP, Washington DC, 1-10.

Borduin, C.M., y Schaeffer, C.M. (1998). "Violent Offending Adolescence: Epidemiology, Correlates, Outcomes, and Treatment", en Delinquent Violent Youth. Theory and Interventions (Edit. Gullotta, Adams y Montemayor). Advances in Adolescent Development, Vol. 9, Sage Publications, Thousand Oaks, CA. 98-128.

Boys, A.; Marsden, J.; Fountain, J.; Griffiths, P.; Stillwell, G., \& Strang, J. (1999). "What influences young people's use of drugs? A qualitative study of decision making. Drugs: education, prevention and policy", 6(3) 373-388.

Bronfenbrenner, U. (1979). The ecology of Human Development. Cambridge, Harvard University Press. (Trad.Cast.): La ecología del desarrollo humano. Barcelona, Ediciones Paidós, 1987).

Brook, D.W.; Brook, J.S.; Zohn, R.; De la Rosa, M.; Montoya I.D., \& Whiteman M. (2003). "Early Risk Factors for Violence in Colombian Adolescents". Am J Psychiatry $160,1470-1478$.

Browning, K., \& Huizinga, D. (1999). "Highlights of Findings from the Denver Youth Survey". En Fact Sheet, April, NN 106, Washington DC. Available in: http://www.ojjdp. ncjrs.org.

Browning, K.; Thornberry, T.P.; \& Porter, P.K. (1999). "Highlights of Findings from the Rochester Youth Development Study". En Fact Sheet, April, № 103, OJJDP, Washington DC. Available in: http://www.ojjdp.ncjrs.org.

Burns, B.J.; Howell J.C.; Wiig, J.K.; Augi-Meri, L.K.; Welsh, B.C., Loeber, R.; \& Petechuk, D. (2003). "Treatment, Services, and Intervention Programs for Child Delinquents". En Child delinquency. Bulletin Series, March, OJJDP, Washington DC. 1-15.

Castillo, M. (2006). "El comportamiento agresivo y sus diferentes enfoques". En Revista de la Universidad Simón Bolívar, 9, 15, 66-170.

Catalano, R.F., \& Hawkins D.J. (1996). "The social development model: A theory of antisocial behavior", en Delinquency and crime: Current theories (ed. Hawkins).Cambridge University Press, New York, 149-197.

Catalano, R.F.; Loeber R., \& Mckinney, K.C. (1999). "School and Community Interventions to Prevent Serious and Violent Offending", en Juvenile Justice Bulletin, October, OJJDP, Washington DC. 1-11.

Farrington, D. (1992). "Implicaciones de la investigación sobre carreras delictivas para la prevención de la delincuencia", en La reeducación del delincuente juvenil. Los programas de éxito (Dir. Garrido y Montoro). Tirant lo Blanch, Valencia, 127-154.

Farrington, D. (1997). "Human Developmentand Criminal Careers". En The Oxford Handbook of Criminology, 2. ${ }^{a}$ ed. (edit. Maguire, Morgan y Reiner). Clarendon Press, Oxford, 361-408.

Feld, B.C. (1999). Bad Kids. Race and the Transformation of the Juvenile Court, Oxford University Press, New York.

Ferrer, X., \& Ayneto, X. (1991). "Nuevos métodos en la formación de padres y madres para la prevención del abuso de drogas". Santa Cruz de Tenerife: XIX Jornadas Nacionales de Sociodrogas y alcohol. 
Fishbei, M., \& Ajzen, I. (1980). Belief, attitude, intention and behavior. An introduction to theory and research. Reading, M.A., Addison-Wesley.

Gómez-Reino, I.; Ferreiro, M.D.; Domínguez, M.D., \& Rodríguez, A. (1995). "Consumo de alcohol en adolescentes: Relación con los niveles de adaptación social y familiar". Psiquis, 16(4), 129.

Gonzales, L. (2012). Violencia social, prevención de la violencia y escuela. Editorial Universidad Don Bosco.

Gutiérrez, J.R. (2012). "La violencia social delincuencial asociada a la salud mental en los salvadoreños". Universidad Tecnológica de El Salvador: Tecnoimpresos, San Salvador.

Gutiérrez, J.R.; Sánchez, M. \& Martínez, R.S. (2013). "Construcción, diseño y validez de instrumentos de medición de factores psicosociales de violencia juvenil". Universidad Tecnológica de El Salvador: Tecnoimpresos, San Salvador.

Hawkins, D.; Lishner, D., \& Catalano, R. (1985). "Childhood predictors and the prevention of adolescent substance abuse". En Jones, C.J., y Battjes, R. (eds.). Etiology of drug abuse: Implications for prevention (pp. 75-126). Rockville: National Institute on Drug Abuse.

Hawkins, J.D.; Herrenkohl, T.; Farrington, D.P.; Devon-Brewer, R.; Catalano, F.; Harachi, T.W., \& Cothern, L. (2000). "Predictors of Youth Violence". En Juvenile Justice Bulletin, April, OJJDP, Washington, DC. 1-10.

Hawkins, J.D.; Michael, W.A.; \& Catalano, R.F. (1995). "Preventing Substance Abuse", en Building a Safer Society. Strategic Approaches to Crime Prevention (edit. Tonry y Farrington). Crime and Justice, Vol. 19, The University of Chicago Press, Chicago, 343-427.

Howell, J.C. (1997). Juvenile Justice \& Youth Violence, Sage, Thousand Oaks, CA.

Huizinga, D.; Loeber, R.; Thornberry, T.P., \& Cothern, L. (2000). "Co-occurrence of Delinquencyand Other Problem Behaviors". En Juvenile Justice Bulletin, November, OJJDP, Washington, DC. 1-8.

Kaleel, A.; Justicia, F.; Benítez, J., \& Pichardo, M. (2007). "Incidencia de los malos tratos entre iguales en alumnos de Hebrón (Palestina)". En Revista del colegio de psicología de Andalucía Occidental y la Universidad de Sevilla. Apuntes en psicología, 25(2), 175-184.

Kaplan, H.B. (1996). "Empirical validation of the applicability of an integrative theory of deviant behavior to the study of drug use". Journal of Drug Issues, 292, 345-377.

Kazdin, A.E. \& Buela-Casal, G. (2001). Conducta antisocial. Evaluación, tratamiento y prevención en la infancia y adolescencia, Pirámide, Madrid.

Laespada, T.; Iraurgi, J., \& Arostegi, E. (2004). "Factores de riesgo de protección frente al consumo de drogas: hacia un modelo explicativo del consumo de drogas en jóvenes de la CAPV". Recuperado de:http://www. izenpe.com/s154812/es/contenidos/informacion/ publicaciones_ovd_inf_txostena/es_9033/adjuntos/ informe_txostena14.pdf

Lawrence, R. (1998). School Crime and Juvenile Justice, Oxford University Press, New York.

Leukefeld, C.G.; Logan T.K.; Clayton R.R.; Martin, C.; Zimmerman, R.; Cattarello, A.; Milich, R., \& Lynam, D. (1998). "Adolescent Drug Use, Delinquency, and Other Behaviors". En Delinquent Violent Youth. Theory and Interventions (Edit. Gullotta, Adams y Montemayor). Advances in Adolescent Development, Vol. 9, Sage Publications, Thousand Oaks, CA. 98-128.

Lipsey, M.W., \& Derzon, J.H. (1998). "Predictors of violent or serious delinquency in adolescence and early adulthood". En Serious and Violent Juvenile Offenders: Risk Factors and Successful Interventions (Edit. Loeber y Farrington). Sage Publications, Thousand Oaks, CA. 86-105.

Lizotte, A., \& Sheppard, D. (2001). “Gun Use by Male Juveniles: Research and Prevention". En Juvenile Justice Bulletin, July, OJJDP, Washington, DC. 1-11.

Luengo-Martín, M.A. \& Tavares-Filho, E.T. (1997). "La estructura de los valores en delincuentes y no delincuentes: un análisis comparativo en los adolescentes marginados en Manaus". XXVI Congreso Iberoamericano de Psicología; Sao Paulo, Brasil.

Malvaceda-Espinoza, E. (2009). "Análisis psicosocial de la violencia entre el conflicto y el desarrollo social", Cuad. Difus. 14, p. 26.

Mendoza, M.I.; Carrasco, A.M., \& Sánchez, M. (2003). "Consumo de alcohol y autopercepción en los adolescentes españoles". Intervención Psicosocial, 12 (19), 95-111.

Moncada-Bueno, S. (1997). "Factores de riesgo y de protección en el consumo de drogas". En Varios: Prevención de la drogodependencia. Análisis y propuesta de ac- 
tuación. Madrid. Ministerio del Interior. Delegación del Plan Nacional sobre drogas. 85-104.

Murcia, H.; Reyes, A.; Gómez, L.; Medina, F.; Paz, B. \& Fonseca, P. (2007). "Caracterización familiar de escolares que presentan comportamiento hostil-agresivo en escuelas públicas y privadas del distrito central". Revista de la Facultad de Ciencias Médicas de la Universidad Autónoma de Honduras. Suplemento N. ${ }^{\circ} 1$.

Orantes, L.F. (2012). "Estado de adaptación integral del estudiante de educación media de El Salvador". Universidad Tecnológica de El Salvador: Tecnoimpresos, San Salvador.

Organización Mundial de la Salud (OMS). (2003). "Informe mundial sobre la violencia y la salud". Washington, DC: Autor

OMS (2003). "Informe mundial sobre la violencia y la salud". Washington, DC: Autor Osterlind, S.J. (1989).

Organización Panamericana de la Salud/Organización Mundial de la Salud (OPS/OMS) (2002). Informe Mundial sobre la violencia y salud. Washington, DC.

OPS/OMS (2006). "Estado del arte de los programas de prevención de la violencia en jóvenes. Basados en el trabajo con la comunidad y la familia, con enfoque de género". Washington, DC: Autor.

Parks, G. (2000). "The High/Scope Perry Preschool Project". En Juvenile Justice Bulletin, October, OJJDP, Washington DC. 1-7.

Pons-Diez, J., \& Berjano-Peirats, E. (1999). "El consumo abusivo de alcohol en la adolescencia. Un modelo explicativo desde la psicología social". Madrid: Plan Nacional sobre Drogas.

Programa de las Naciones Unidas para el Desarrollo, El Salvador (PNUD). (2013). Informe Regional de Desarrollo
Humano 2013-2014. "Seguridad ciudadana con rostro humano: diagnóstico y propuestas para América Latina". San Salvador: Programa de las Naciones Unidas para el Desarrollo.

Romero, E. (1996). La prevención de la conducta antisocial: Un análisis de las variables de personalidad. Universidad de Santiago de Compostela: Tesis Doctoral.

Schweinhart, L.J. (2003). "Validity of the High/Scope Preschool Education Model". En High/Scope Educational Research Foundation, February, 21, Ypsilanti, MI, 1-21.

Seydlitz, R., \& Jenkins, P. (1998). "The influence of Families, Friends, Schools, and Community on Delinquent Behavior". En Delinquent Violen Youth. Theory and Interventions (Edit. Gullotta, Adams y Montemayor). Advances in Adolescent Development, Vol. 9, Sage Publications, Thousand Oaks, CA, 53-97.

Smith, M.J., \& Scott, R.D. (1993). "Reasons for drinking alcohol: Their relationship to psychological variables and alcohol consumption". The International Journal of the Adicctions, 28(9), 881-908.

Unicef (2006). El Estado Mundial de la Infancia. Excluidos e invisibles. Autor. Unicef House, 3 UN Plaza, Nueva York, NY, 10017, USA. Recuperado de: http://www.unicef. org/spanish/sowc06/pdfs/sowc06_fullreport_sp.pdf

Wasserman, G.A.; Keenan, K.; Tremblay R.E.; Coie, J.D.; Herrenkohl, T.l; Loeber, R., \& Petechuck, D. (2003). "Risk and Protective Factors of Child Delinquency". En Child Delinquency. Bulletin Series, April, OJJDP, Washington DC. 1-14.

Zúñiga, A. y Bermudez, M.P. (2010). “Efectos de la separación de los padres sobre la adaptación de la conducta, el autoconcepto y la ansiedad en adolescentes salvadoreños". Revista Salvadoreña de Psicología, Vol. 1, 52-65. 